九州大学学術情報リポジトリ

Kyushu University Institutional Repository

BLOW FLIES OF MEDICAL IMPORTANCE IN NEW GUINEA, BISMARCK ARCHIPELAGO AND BOUGAINVILLE ISLAND (DIPTERA : CALLIPHORIDAE) PART I. GENERA CALLIPHORA, TAINANINA, POLLENIOPSIS AND MELINDA

Kurahashi, Hiroshi

https://doi.org/10.5109/2485

出版情報: ESAKIA. 24，pp.5-18，1986-01-31. Entomological Laboratory，Faculty of Agriculture， Kyushu University

バージョン：

権利関係 : 


\title{
BLOW FLIES OF MEDICAL IMPORTANCE IN NEW GUINEA, BISMARCK ARCHIPELAGO AND BOUGAINVILLE ISLAND (DIPTERA : CALLIPHORIDAE) PART I. GENERA CALLIPHORA, TAINANINA, POLLENIOPSIS AND MELINDA"
}

\author{
HiRomu KuRAHASHI \\ Department of Medical Zoology ${ }^{2)}$, Faculty of Medicine, Tokyo \\ Medical and Dental University, Tokyo 113, Japan
}

\begin{abstract}
One hundred twelve species (including 23 new species and 5 unrecorded species) of the genera Calliphora, Tainanina, Onesia, Polleniopsis, Melinda, Hemipyrellia, Lucilia, Phumosia, Euphumosia, Dexopollenia, Bengalia, Chrysomya, Rhinia, Chlororhinia, Stomorhina, Idiella, Metallea, Platytropesa, Stilbomyella and Paramenia are recognized from New Guinea, Bismarck Archipelago and Bougainville Island as a result of field works supported by Grants-in-Aid for Overseas Scientific Survey from the Japan Ministry of Education, Science and Culture since 1973.

This paper, as the first one of my report, deals with Calliphora, Tainanina, Polleniopsis and Melinda and presents a key to all the 20 genera mentioned above. Three new species, Calliphora (Paracalliphora) kanoi, Melinda muficornis and Melinda flavipennis are described.
\end{abstract}

\section{Introduction}

The calliphorid flies collected during expeditions to Papua New Guinea and the South Pacific Islands (Tokyo Medical and Dental University Overseas Scientific Research Project, 1973, 1975, \& 1977 ; Kyushu University Overseas Scientific Research Project, 1982 \& 1984) were studied. I also had an opportunity to examine some specimens on loan from Bishop Museum, Honolulu (Bishop) : British Museum (Nat. Hist.), London (BMNH) ; Department of Primary Industry, Konedobu, PNG (DPI) ; and Forest Research Station, Bulolo, PNG (FRS). These specimens enabled me to study the Calliphoridae from New Guinea, the Bismarck Archipelago and Bougainville Island,

This study was supported by the Grants-in-Aid for Overseas Scientific Survey, Ministry of Education, Science and Culture, in 1973 and 1977 to Tokyo Medical and Dental University (Prof. R. Kano) and in 1982 and 1984 to Kyushu University (Prof. Y. Hirashima). The publication of this paper was supported by the Grant No. 60043050 (Prof. Y. Hirashima).

'hief : Prof. R. Kano. Correspondence : H. Kurahashi, Department of Medical Entomology, National Institute of Health, 2-10-35, Kamiosaki, Shinagawa-ku, Tokyo 141, Japan. 
and to summarize the calliphorid fauna of the area for the first time. A total of 112 species, including the previously recorded ones, are represented in the fauna. Twentythree of these species are new to science and 5 are recorded for the first time from the area.

Among the previous works, the following two and some others (Theowald 1957, 1959) are very useful to study the blow flies of New Guinea and its adjacent islands. An excellent revision on the Ameniine Calliphoridae (Crosskey 1969) reported 7 species from New Guinea and the Bismarck Archipelago. Torgerson and James (1967) also published an encouraging paper on the genus Euphumosia and listed 32 species endemic in New Guinea, Ambon, Ceram and Northern Territory of Australia. James (1971 a, b) and Kurahashi $(1971,1972,1978,1980,1984)$ described or redescribed the chrysomyine and calliphorine blow flies from New Guinea and the Bismarck Archipelago.

The blow fly fauna in New Guinea and its adjacent islands is characterized by the dominance of endemic forms (77 spp., $69 \%$ ). The genus Euphumosia is certainly peculiar to this area and consists of $35 \mathrm{New}$ Guinea species. The genus $\mathbf{O}$ nesia also contains 11 endemic species. Seven species of the New Guinea Ameniinae have never been recorded from other than New Guinea. One or a few endemic species are found in each of the following genera : Calliphora, Polleniopsis, Melinda, Lucilia, Hemipyrellia, Phumosia, Dexopollenia, Bengalia, Chrysomya, Stomorhina, Idiella and Metallea. The Indo-Australian (21 spp., $19 \%$ ) and Australian (12 spp., $10 \%$ ) make up about $30 \%$ of the total blow fly fauna in New Guinea and its adjacent islands. Luciliacuprina (Wiedemann) and R hinia apicalis (Wiedemann) are known to be pan-tropical (1\%). Hemipyrellialigurriens (Wiedemann) widely distributed in the Indo-Australian area and partly invading the Palaearctic Asia such as China, Korea and Japan. Chrysomya megacephala (Fabricius) and Ch. bezziana Villeneuve are certainly common in the IndoAustralian area, but the former was recently introduced into the Afrotropical and Neotropical Regions. These two flies are well known to be harmful and filth pests and they are so called "the Oriental Latrine Fly" and "the Old World Screw Worm Fly", respectively.

BLow FLIES OF MEDICAL IMPORTANCE

The blow flies are considered as one of groups of medical importance. Some species have been known as myiasis producers and their larvae normally developed in carcases, sometimes become facultative parasites in traumatic lesions. Chrysomya bezziana, the Old World Screwworm Fly, is an obligatory parasite which causes traumatic myiasis of man and domestic animals. New Guinea is a screwworm-ridden country. Maggots of this species infest all parts of soft and mucous tissues of human beings such as the nose, mouth, ear, axilla, orbits and the genital organs of male and female. Such domestic animals as cattle, carabao, goat, horse and sheep are also attacked by this screwworm and develop skin wounds and injured parts of the body resulting from faulty dehorning.

Another species called the Oriental Latrine Fly, Chrysomya megacephala, which is 
closely related to Ch. bezziana, is a feces and carrion breeder and very common around human dwellings and frequent foods in markets. The omnivorous and promiscuous feeding habits enable these flies to be potential vectors of a number of diseases of man and animals. Pathogenic organisms and other helminthic parasites are mechanically transferred from an infected source to human food through the appendages, body hairs or bristles of blow flies which feed or congregate on infected filth, such as human excrement and sewage, and through vomit drop or regurgitation habits and fecal droppings. They may thus become of considerable importance during epidemics of intestinal diseases such as typhoid, cholera and amoebiasis. The origin and establishment of the synanthropy of Ch. megacephala has been studied in Wau Ecological Institute, Papua New Guinea (Kurahashi 1982b, 1984) and the details will be discussed further in separate pepers, including the ecological observations made on the last survey trip.

\section{Key to the Genera OF New Guinea Calliphoridae}

1. Stem vein setulose above

- Stem vein bare

2. Anterior lappet of metathoracic spiracle with a conspicuous backwardly-directed tuft of long hairs; postscutellum forming a definite convex swelling which is microrugose and

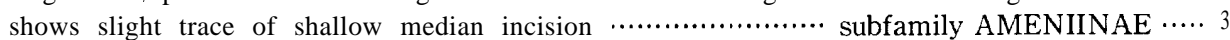

- Anterior lappet of metathoracic spiracle bare or at most with a very few small inconspicuous hairs ; postscutellar region not at all convex or at most with rudimentary trace

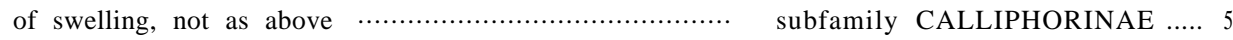

3. Head with a very strong facial carina separating antennae; hind tibia with apical $p v$;

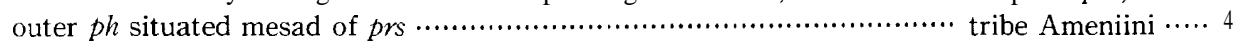

- Head without a facial carina ; hind tibia without definite apical $p v$; outer $p h$ situated laterad of $p r s$.............................................. tribe Parameniini, genus Paramenia

4. Fore tibia with $2-3$ small but distinct $p d$; facialia with fine setulae extending more than $1 / 2$ or about $1 / 2$ way up their length, in profile reaching far beyond level of apex of

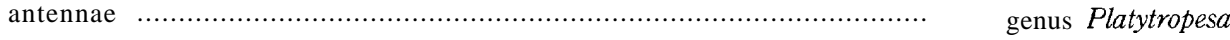

- Fore tibia without $p d$; fine hairs above vibrissae confined to lower $1 / 4$ of facialia, in profile only extending at most a little beyond apex of antennae ............. genus Stilbomyella

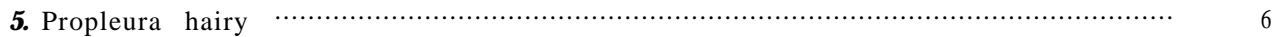

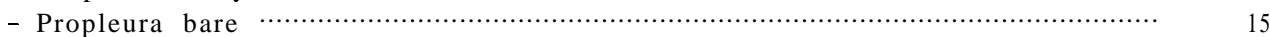

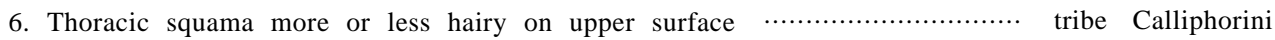

- Thoracic squama quite bare on upper surface …............................................ 7

7. Posterior part of suprasquamal ridge with a tuft of black setulose erect hairs on a small

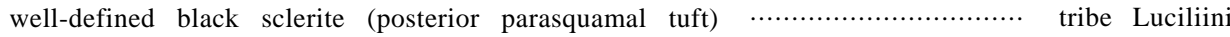

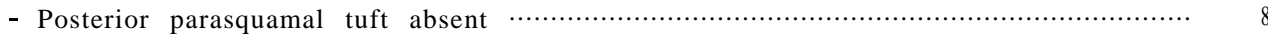

8. Anterior part of suprasquamal ridge bare; distance between right and left of presutural ac rather large, as shown in Fig. la ; mosothoracic spiracle rather large, remarkably

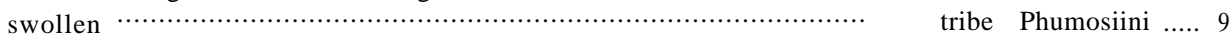

- Anterior parasquamal tuft present, if not so, a distance between right and left of presutural ac small as shown in Fig. lb ; mesothoracic spiracle smaller, not swollen......

9. Supraspiracular convexity clothed with long, upstanding, fine hairs …......... genus Phumosia Supraspiracular convexity bare or pubescent genus Euphumosia 
10. Supraspiracular convexity clothed with long, upstanding, fine hairs …..... genus Hemipyrellia

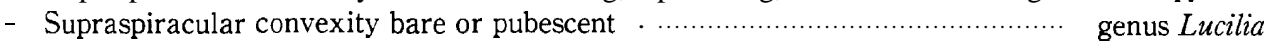

11. Presutural ac usually 1 , rarely absent ; facial carina distinctly developed $\cdots$ genus Polleniopsis

- Presutural ac 2, sometimes 3 ; facial carina absent …............................................ 12

- Presutural ac 1-2, anterior one usually fine, sometimes absent ; eyes covered with hairs ; epistome remarkably projecting ; facial carina absent ; ac1-2+2...................

subgenus Calliphora (Papuocalliphora)

12. Thoracic squama quite bare on dorsal surface ; anterior parasquamal tuft developed ..... genus Melinda

- Thoracic squama more or less hairy on dorsal surface, if not so, anterior parasquamal portion bare

13. Male frons very broad ; eyes dichoptic in $\sigma^{7}$ and $q$; 3rd antennal segment elongate, more than 4 times as long as 2 nd ; presutural $i a$ absent ........................ genus Tainanina

- Eyes holoptic in $\sigma$ and dichoptic in $q$; length of 3rd antennal segment variable; presutural ia present or absent

14. Genae bright orange to yellow in ground colour, more or less yellowish-dusted ; distance between right and left of presutural ac rather large, as shown in Fig. la ; body rather large ............................................................. subgenus Calliphora (Paracalliphora)

- Genae fuscous in ground colour, as a rule, densely gray or yellowish-gray dusted; distance between right and left of presutural ac small as shown in Fig. lb ; body usually small to medium in size, sometimes rather large …................................... genus Onesia

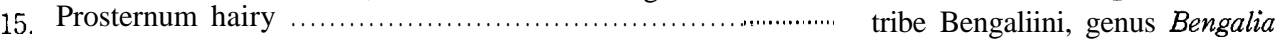

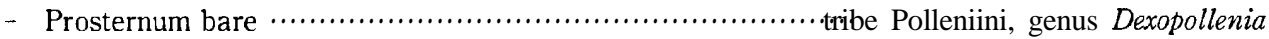

16. Thoracic squama with hairs on upper surface ; subcostal sclerite setulose; pre-alar knob usually setulose (if bare, then metallic blue and yellow flies as in Eucompsomyia

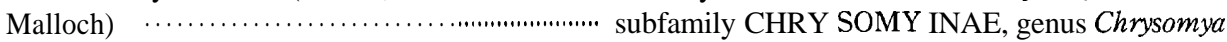

- Thoracic squama bare above ; subcostal sclerite with fine pubescence ; pre-alar knob

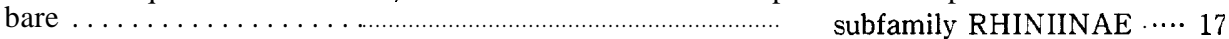

17. Arista pectinate ; ac and dc not distinguishable from the general ground hairs, the prescutellars at most weakly developed ; suprasquamal ridge bare ……. tribe Rhiniini $\cdots \cdots 18$

- Arista pubescent or plumose, not pectinate …...........................tribe Cosmini .... 21

18. Outer $p h$ absent …........................................................... genus Chlororhinia

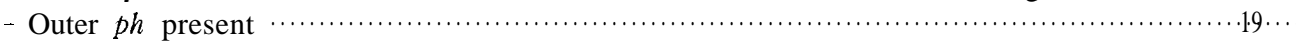

19. Hind tibia without a conspicuous row of ad, but with 2-3 ad as long as or longer than the tibial diameter; $R_{5}$ open ; body slender, parallel-sided ; abdomen mostly

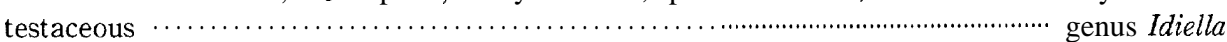

- Hind tibia with a conspicuous row of subequal ad, longer than the normal ground hairs, sometimes 2-3 rather strong ad developed among them ; front tibia with $1 p v$; body rather stout with ovate abdomen and usually of a dark colouration …......................... 20

20. $\mathrm{R}_{5}$ petiolate ; sternopleura glossy, without dusting ; mesopleura without setigerous spots ; mesopleural row of bristles incomplete ; legs entirely yellow ; tergite $1+2$ with

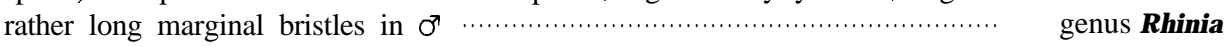

- $R_{5}$ open in the wing margin or closed; if petiolate then the sternopleura are heavily dusted genus Stomorhina

21. Arista almost bare, only microsopically haired ; propleura hairy ……........... genus Metallea - Arista long-plumose ; propleura bare ; no record from Australian Region genus Isomyia

\section{Genus Calliphora Rob.-Desvoidy}

Calliphora Rob.-Desvoidy, 1830. Essai Myod. : 433. Type-species : Muscavomitoria Linné, 1758. 


\section{BLOW FLIES OF NEW GUINEA AND ADJACENT ISLANDS}

\section{Subgenus Papuocalliphora Kurahashi}

Papuocalliphora Kurahashi, 1971. Pac. Insects 13 : 167. Type-species : Calliphora toxopeusi Theowald, 1957.

\section{Calliphora (Papuocalliphora) toxopeusi Theowald}

Calliphora toxopeusi Theowald, 1957. Nova Guinea, new ser. 8:158.

Calliphora (Papuocalliphora) toxopeusi : Kurahashi, 1971. Pac. Insects 13: 167.

Length : 7.5-11.0 mm.

SPeCIMENS EXAMINED. NEW GUINEA (PNG) :30 39 \% , Mt. Hagen, 2,910 m, 12. II. 1978, Kano (TMDU) ;2 635 \&, Mt. Kaindi, Wau, 10-13. I. 1974, 29. I. 1985, Shinonaga \& Kurahashi (TMDU \& KU) ; 9 \&, Daulo Pass, 2,467 m, nr Goroka, 1. II. 1978, Kano (TMDU) ; 2 q, Mt. Ialibu-Mt. Giluwe, 2,000 m, 2. II. 1978, Kano (TMDU) ; 3 , Margarima Farm, S. Highland, 2,000 m, 5. II. 1978, Kano (TMDU) ;1 $q$, Wak River, Margarima, 2,100 m, 5. II. 1978, Kano (TMDU) ; 13 q, Tari Gap, 2,500 m, 3. II. 1978, Kano (TMDU) ; $10 \subsetneq$, Mt. Wilhelm, 3,600 m, Chimbu Dist., 1. VIII. 1982, Ismay (DPI) ; 9 , Alpinegrass Country, Mt. Strong, Central Dist., 11,700 ft, 30. VIII. 1963, ? (DPI);1 q, Mt. Scratchley, 11,000 ft, Central Dist., 7-11. VI. 1971, Wilkins (DPI). BOUGAINVILLE I. :1 , Mt. Balbi, 2,000-2,400 m, 1-7. III. 1968, Straatman (Bishop).

Bionomics. Larviparous. Adults are found in highlands above $2,000 \mathrm{~m}$ alt.

Distribution. New Guinea (PNG) and Bougainville I.
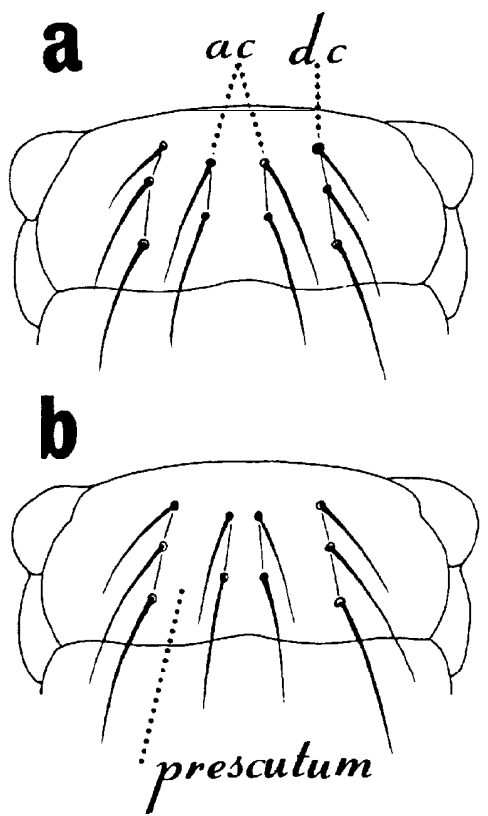

Fig. 1. Prescutal chaetotaxy: a, Calliphora-type ; b, Onesia-type. 
Subgenus Paracalliphora Townsend

Paracalliphora Townsend, 1916. Can. Ent. 48 : 151. Type-species : Calliphora oceaniae Rob.- Desvoidy, 1830 .

\section{Key to THE SPECIES OF Paracalliphora}

1. Basicosta yellowish brown ; abdomen honey yellow on at least sides and venter, always

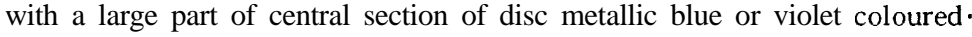

C. porphyrina Kurahashi

- Basicosta black ; abdomen entirely metallic blue, sometimes blackish shining, without conspicuous dusting

2. Front coxa with some yellow hairs on anterior surface at base; occipital dilatation behind genae usually yellowish orange ; face reddish brown …......... C. papuensis Kurahashi Front coxa black haired ; occipital dilatation just behind genae fuscous ; face submetallic, rarely reddish above epistome in $\sigma^{7}$

C. kanoi sp. nov.

\section{Calliphora (Paracalliphora) porphyrina Kurahashi}

Calliphora (Paracalliphora) porphyrina Kurahashi, 1971. Pac. Insects $13: 181$.

Length : $5.0-8.5 \mathrm{~mm}$.

SPECIMENS EXAMINED. NEW GUINEA (PNG) :2 $q$, Gumi, logging area, 6,000 ft, 13. IX. 1984, Roberts (FRS) ;13 ơ 56 \&, Mt. Kaindi, Wau, 26-29. XII. 1973, 5-17. I. 1974, 10 13. I. 1974, 29. I . 1985, Kano, Shinonaga, Shima \& Kurahashi (TMDU \& KU) ; $10^{\text {' }}$, Mendi-Nipa, 2,200 m, 3. II. 1978, Kano (TMDU) ; 10 ơ 5 q, Myola 2, Oro Prov., 20. IV4. V. 1984, 2,080-2,200 m, Ismay (DPI) ;10 1 1 , Mt. Manki, 1,500 m, Bulolo, Morobe Prov., 31. I. 1985, Kurahashi (KU) ; 3 q, Goroka town, 31. I. 1978, Kano (TMDU) ; 9 q, Mahkam River, nr Lae, 29. I. 1978, Kano (TMDU) ; 4 + , Zenag, 1,130 m to Gurokor, 800 m, 29. I. 1978, Kano (TMDU) ;1 9 , nr Nipa, 1,300 m, 3. II. 1978, Kano (TMDU) ;1 Kaunan, 1,900 m, W. Highland, 13. II. 1978, Kano (TMDU).

Bionomics. Adults are attracted to decaying animal matter and human feces. Larviparous.

Distribution. New Guinea (PNG).

\section{Calliphora (Paracalliphora) papuensis Kurahashi}

Calliphora (Paracalliphora) papuensis Kurahashi, 1971, Pac. Insects $13: 171$.

Length : 7.0-8.5 mm.

Specimens eXAmined. NEW GUINEA (PNG) : $10^{7} 3$, Gumi, logging area, 5,5006,000ft, 11-13. IX. 1984, Roberts (FRS) ;10 , Myola, N. Prov., 2,200 ft, 2. XI. 1984, Roberts (FRS) ;17 đ'1 +, Iora Creek, nr Myola 2, 2,080 m, 20. IV-3. V. 1984, Ismay (DPI) ; $2 \sigma^{\top} 1$ ㅇ, Daulo Pass, 2,400 m, 40 km W of Goroka, East H. Dist., 29-30. VII. 1982, Ismay (DPI) ;1 10', Tambul, 2,200 m, West H. Dist., 12. VIII. 1982, Ismay (DPI) ;1 3 ' 3 , Mt. Kaindi, Morobe Prov., 14. IX. 1981, Ismay (DPI) ; 1 , Sinsibai, 2,400 m, W. H. Prov., 6. V. 1984 (DPI) ;1 \&, Kakaitomba, 2,570 m, 26 km W of Mt. Hagen, W. H. Dist., 
10. VIII. 1982, Ismay (DPI) ;10 , Aiyura, 10. II. 1956, J. H. B. (DPI) ; 10, Daulo Pass, 8,175-8,300 ft, Bismarck Range, E. H. Dist., ? (DPI) ; 1 O’ $^{\prime}$ \&, Mt. Kaindi, 2,362 m, Wau, Morobe Prov., 29. I. 1985, Kurahashi (KU) ; 202 \& , Tomba, ca 1,800 ft, $25 \mathrm{~km} \mathrm{~W}$ of Mt. Hagen, W. Highlands, 5. VI. 1965, Crosskey (BMNH) ;10ْ 39, Wau, 2,500-4,000 ft, Morobe Dist., 14-23. V. 1965, Crosskey (BMNH) ;10̛ 49, Daulo Pass, 8,175ft, 25 km W of Goroka, 31. V. 1965, Crosskey (BMNH) ;1 $9, \mathrm{~S}$ side of Mt. Abilala, 8,000 ft, Finisterre Mts., Madang Dist., 17. XI. 1964, stn. No. 97 (BMNH) ;1 , Mt. Tafa, 8,500 ft, Papua, II. 1934, Cheesman (BMNH-1984-321) ; 39, Yemi Vill., Goilala, C. D. of Papua, 14. X. 1963, Smee, Ex. Coll. Dept. Agr. P. M. Nos. 5065, 5066, 5068 (BMNH) ; 190 6252 q, Mt. Kaindi, Wau, 10-13. I 1974, 26-29. XII. 1974, Kano, Shinonaga \& Shima (TMDU) ; 22061 , Mt. Ialibu-Mt. Giluwe, 2,000 m, 2. II. 1976, Kano (TMDU) ; 5 o 15 ㅇ , Mendi to Mt. Hagen, 2,100 m, 11. II. 1976, Kano (TMDU) ;60 49, Tari Gap, 2,500 m, 3. II. 1978, Kano (TMDU) ; $50^{7} 5 q$, Mendi-Nipa, 2,200 m, 3. II. 1978, Kano (TMDU) ; 13 ơ 129, Margarima Farm, S. Highland, 2,000 m, 5. II. 1978, Kano (TMDU) ; 305 , Daulo Pass, 2,467 m, nr Goroka, 1. II. 1978, Kano (TMDU) ; 305 \&, Wak River, Margarima, 2,100 m, 5. II. 1978, Kano (TMDU) ;1 ơ 8 9 , nr Margarima, 2,000 m, 3. II. 1978, Kano (TMDU) ; 79, Mt. Hagen, 2,910 m, 12. II. 1978, Kano (TMDU) ; 7 , Kaunan, 1,900 m, Mt. Hagen, W. Highland, 13. II. 1978, Kano (TMDU) ;1 9 , Aiyura, 1,650 m, 14. II. 1978, Kano (TMDU).

Bionomics. Adult are attracted to decaying animal matter and human feces. Larvae breed in human feces. Larviparous.

Distribution. New Guinea (PNG).

\section{Calliphora (Paracalliphora) kanoi, new species Fig. 2}

○. Head : eyes bare, holoptic, narrowly separated on frons by a distance equal to width of ocellar triangle ; frons index 0.05-0.07; frontal stripe black, widened anteriorly and posteriorly, not obliterated at narrowest point ; frontalia dull yellowish-gray to gray-dusted, darkened toward vertex, with fine, rather long black setulae anteriorly, provided with about 10 pairs of ori; face blackish, slightly gray-dusted, without median carina; parafacialia yellowish-gray dusted, with rather long, fine black setulae above ; facialia yellowish brown, with black setulae on lower $1 / 2$ from vibrissae to antennal bases ; medianae and vibrissaria reddish brown, bare ; vibrissae strongly developed ; genae and postgenae orange, yellowish-gray to golden-dusted, genae are clothed with black hairs except for yellowish some along peristomal margins ; postgenae entirely clothed with yellow hairs ; occipital dilatation behind upper comer of genae black, yellowish-gray dusted, clothed with yellow hairs ; occiput black, dull yellowish-gray dusted, clothed with yellow hairs except for a row of postorbital bristles ; epistome distinctly projecting forward, fuscous brown ; 3rd antenna1 segment largely blackish except for ventral surface of basal portion and joint of 2nd and 3rd segment reddish, slightly more than $3.5 \mathrm{x}$ as long as 2nd ; 2nd segment fuscous brown; arista black, long-plumose on basal 2/3; palpi light brown. Thorax: black, with bluish tinge, entirely with thin covering of white dusting, without any remarkable marking on dorsum ; scutellum, humeri and postalar calli concolorous with scutum; prosternum blackish haired ; propleura with yellowish brown hairs, other pleural sclerites covered with black hairs; hypopleural bristles black; pleurotergite with patch of black setulae ; supraspiracular convexity pubescent, without setulose hairs ; postalar declivity with tuft of blackish hairs on central portion; suprasquamal ridge with anterior parasquamal tuft of more than 10 fine black hairs ; tympanic tuft not developed, only a few black hairs are present; mesothoracic spiracle black, metathoracic one blackish. Chaetotaxy; ac $2+3$, dc $2-3+3$, ia $0+2, h 2-4$, ph 3 , prs 1 , sa 
$3,+2$ fine interstitials, pa 2 , st $2+1$, sc $3+1, p p 2-3$, pst 2 . Wings: hyaline, slightly infuscated at base ; veins blackish ; epaulet and basicosta black ; subcostal sclerite fuscous black, with blackish brown pubescence; node of 2nd and 3rd longitudinal veins with several black setulae above and below, the setulose hairs are extended to $1 / 2$ way to $\mathrm{r}-\mathrm{m}$ cross vein above ; 4 th longitudinal vein bent with a right angle, but the corner is rounded, section of the 4th vein from bend to wing edge is inflexed; squamae entirely blackish brown, thoracic one is lobulated, almost entirely covered with fine long black hairs on upper surface. Halteres blackish. Legs : black, with black hairs ; front tibia with $1 p$ and 3-5 short ad ; mid tibia with 2-3 ad, $1 p d, 2 p$ and $1 v$; hind tibia with 2-3 ad, $2 p d$ and 2 av. Abdomen : short oval, entirely dark blue, metallic, without pruinosity, blackish haired, the hairs on sternites are rather long and fine ; tergites $1+2$ and 3 with several lateral marginal bristles ;

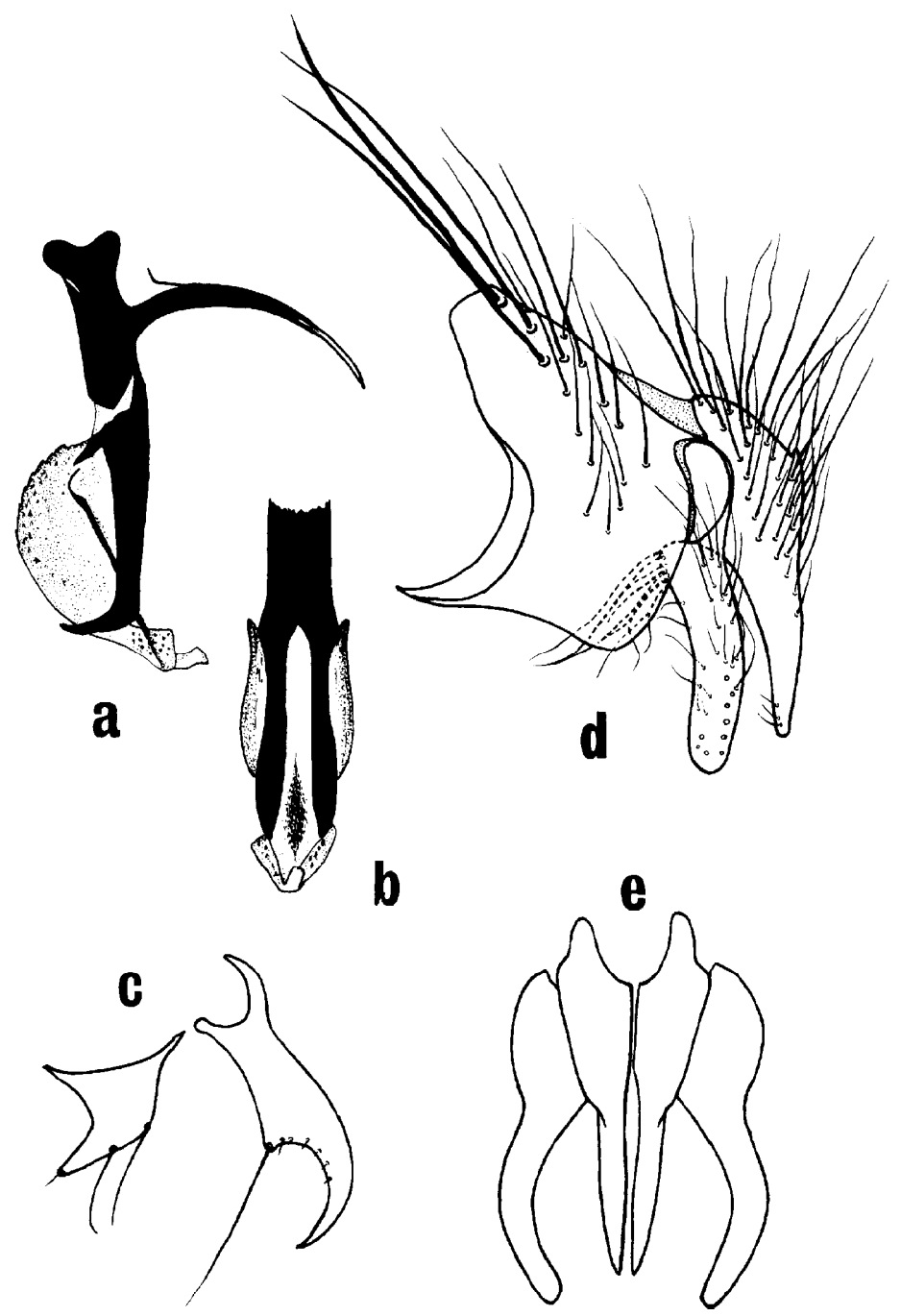

Fig. 2. Calliphora kanoi sp. nov., male hypopygium : a, aedeagus, lateral view ; b, aedeagus without basal portion, posterior view ; c, anterior and posterior parameres, lateral view ; d, epandrium, cercus and paralobus, lateral view ; e, cerci and paralobi, caudal view. 
tergite 4 with complete row of erect marginals ; tergite 5 with erect fine bristles on disc and hind margin. Hypopygium normal in size, $\sigma^{7}$ genitalia as shown in Fig. 2.

\%. Head : eyes separated at vertex by a distance equal to $0.32-0.33$ of head width ; frontal stripe black, slightly narrowed posteriorly, slightly more than $2 \mathbf{x}$ the width of 1 of parafrontalia just in front of anterior ocellus ; parafrontalia dull golden-dusted, darkened toward vertex, rather densely setulose above, provided with ca 8 pairs of ori; ors $2+1 ;$ oc developed ; acoc absent ; ov and iv well developed ; poc parallel or divergent ; occ 1. Legs : hind tibia with 3 ad and 3 pd. Abdomen : dark blue, sometimes with copper, green or purple tinges; tergite 4 without complete row of marginal bristles, only lateral marginals are developed. Ovipositor of moderate length. Otherwise same as for o'.

\section{Length : 7.0-9.5mm.}

Holotype , NEW GUINEA (PNG) : Mt. Ialibu-Mt. Giluwe, 2,000 m, 2. II. 1978, Kano (TMDU). Paratypes : NEW GUINEA (PNG) : 3 ㅇ, same data as holotype ; $1 \sigma^{\top}$, Myola 2, forest, 2,080-2,200 m, Oro Prov., 1. V . 1984, Ismay (DPI) ; 1 , Mt. Giluwe, 2,700 m, S. H. Dist., 11. VIII. 1982, Ismay (DPI) ; 1 , Tambul, 2,200 m, W. H. Dist., 12. VIII. 1982, Ismay (DPI) ; 7 o 5 o , Murmur Pass, 2,760 m, W. H. Dist., 20. X. 1981, Ismay

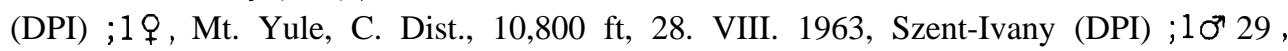
Myiola, N. Prov., 2,200 m, 2. XI. 1984, Roberts (DPI) ;1 'ণ', Kakaitomba, 2,500 m, 26 km W of Mt. Hagen, W. H. Dist., 10. VIII. 1982, Shinonaga (TMDU) ; $17069 \%$, Daulo Pass, Goroka, 2,300 m, 14. VIII. 1982, Shinonaga (TMDU).

The specific name is dedicated to Prof. Dr. R. Kano, chief of the project, who collected this interesting fly from the upper mountain in Papua New Guinea during his survey trip.

TyPE DEPOSITORY. Holotype in Bishop Museum ; paratypes in DPI, FRS, BMNH, KU, National Science Museum, Tokyo (NSMT).

Bionomics. Adults are attracted to decaying meat in native forests. Larviparous. Distribution. New Guinea (PNG).

\section{Genus Tainanina Villeneuve}

Tainanina Villeneuve, 1926, Bull. Ann. Soc. Ent. Belge $66: 217$. Type-species :T. grisella Villeneuve, 1926 (=T. pilisquama (Sen.-White, 1925)).

\section{KeY TO THE SPECIES of Tainanina}

1. External $p h$ usually developed ; presutural dc 2-4; hairs on thoracic squama usually yellow ; squamae and bases of wings yellowish ; larger species, more than $6.5 \mathrm{~mm}$ in length T. javanica Kurahashi

- External $p h$ absent ; presutural dc usually 2 ; hairs on thoracic squama fuscous brown ; wings hyaline ; smaller species, less than $6.5 \mathrm{~mm}$ in length

T. sarcophagoides (Malloch)

\section{Tainanina javanica Kurahashi}

Tainanina javanica Kurahashi, 1978, Pac. Insects $18: 6$. — Inder-Singh et al., 1979, Bull. Tokyo Med. Dent. Univ. $26: 10$.

Length: 6.5-8.5 mm.

SPECIMENS EXAMINED. NEW GUINEA (IRIAN JAYA: INDONESIA) : 19 , Wisselmeren, Enarotadi, 1850 m, 12. VII. -4. VIII. 1962, Sedlacek (Bishop). 
Bionomics. Nothing is known. The type materials were collected in tea plantation along the margin of native rain forest.

Distribution. Malay (Inder Singh et al., 1979), Java and New Guinea (Irian Jaya).

\section{Tainanina sarcophagoides (Malloch)}

Calliphora sarcophagoides Malloch, 1931, Ann. Mag. Nat. Hist. (10) 7 : 192.

Tainanina sarcophagoides : Sen. -White et al., 1940, Fauna Brit. India, Dipt. 6: 110. -James, 1966, Ent. Meddel. 34 : 466. — Kurahashi, 1978, Pac. Insects $18: 4$. — Tumrasvin et al., 1979, Bull. Tokyo Med. Dent. Univ. $26: 248$.

Length : 3.0-6.5 mm.

Specimens examined. NEW GUINEA (IRIAN JAYA: INDONESIA) : $2 \sigma^{\prime}$, Wamena, 1,500-1,700 m, 10-25. II. 1960, Maa (Bishop) ;10', Wisselmeren, Enarotadi, 1850, 12. VII.-4. VIII. 1962, Sedlacek (Bishop). NEW GUINEA (PNG):10', Swart Val., Karubaka, 1,550 m, 8. XI. 1958, Gressitt (Bishop) ;20̛1, Wau, Morobe Prov., 9801250 m, 14. VIII. 1964, 4. V. 1965, 18. X. 1965, Sedlacek (Bishop) ;10’, Hospital Creak, 1,230 m, Wau, 26.VI.1965, Sedlacek (Bishop). NEW IRELAND :20̛', Namatanai, 2123. I. 1974, Shinonaga (TMDU).

Bionomics. Nothing is known.

Distribution. Taiwan, Philippines, Vietnam, Thailand, Borneo, Malay, Lombok, New Guinea, Bismarck Arch. and Solomon Is.

\section{Genus Polleniopsis Townsend}

Polleniopsis Townsend, 1917. Rec. Ind. Mus. 13 : 201. Type-species : Polleniopsis pilosa Townsend, 1917.

Key to the SPECIES of Polleniopsis

1. Presutural ia present ; abdomen black, densely covered with yellowish gray or golden dusting, strongly tessellated ; legs black; facial carina well developed ; external $p h$

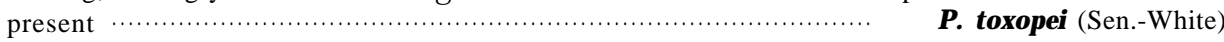

- Presutural ia absent

2. External ph present; abdomen bluish green, densely pruinose ; legs black; posterior part of genae black

P. jamesi Kurahashi

- External $p h$ absent ; abdomen tawny, sometimes semitransparent ; genae yellow

P. fulviventris Kurahashi

\section{Polleniopsis toxopei (Sen.-White)}

Paratricyclea toxopei Sen.-White, 1926, Rec. Ind. Mus. $28: 135$.

Pollenia toxopei : Sen.-White et al., 1940, Fa. Brit. India, Dipt. $6: 122$.

Polleniopsis toxopei : Kurahashi, 1972, Pac. Insects $14: 710$.

Length : 5.0-9.5 mm.

SPECIMENS EXAMINED. NEW GUINEA (IRIAN JAYA: INDONESIA) :107, Waris, 
$\mathrm{S}$ of Hollandia, 450-500 m, 24-31. VIII. 1959, Maa (Bishop). NEW GUINEA (PNG) : 2 $\sigma^{7}$, Mt. Gahavisuka, 6,500 ft, 23. VI. 1985, Roberts (FRS) ; $9 \sigma^{\top} 5$, Wau, 1,200 m, Morobe Prov., 20-26. V. 1962, 5-17. I. 1974, 30. I-2. II. 1985, J. Sedlacek, Shinonaga \& Kurahashi (Bishop, TMDU \& KU) ; $1 \sigma^{\top 1} 1$, Botanical Garden, Lae, 15. II. 1978, 30. I. 1978, Kano (TMDU) ;1 $\sigma^{71}$ \&, Lake Kutubu, S. Highland, 1,000 m, 6-9. II. 1978, Kano (TMDU) ;1 đ̛, Brown River, Port Moresby, 13-14. I. 1978, Kano (TMDU) ; 7 ơ 16 ㅇ, Margarima Farm, S. Highland, 2,000 m, 5. II. 1978, Kano (TMDU) ;10 , Kandan, 1. I. 1960, Brandt (Bishop) ; 1 , Simbai, 1,600-1,800 m, N. E. New Guinea, ? X. 1968, Krauss (Bishop). NEW BRITAIN : 203 \&, Keravat, Rabaul, 25-27. I. 1974, Kano \& Shinonaga (TMDU). NEW IRELAND :2 ơ1 + , Namatanai, 21-23. I. 1974, Shinonaga (TMDU).

Bionomics. Nothing is known.

Distribution. Malay, Borneo, Sumatra, Java, Buru I., New Guinea, Bismarck Arch., Solomon Is. and Admiralty Is.

\section{Polleniopsis jamesi Kurahashi}

Polleniopsis jamesi Kurahashi, 1972, Pac. Insects 14 : 714. -Kurahashi, 1980, Pac. Insects 22 : 171.

Length : 8.5-11.0 mm.

Specimens examined. NEW GUINEA (PNG) :10 , K. Rehder Ptn., Kainantu, 3. VI. 1960, ? (DPI) ;1 , Aiyura, E. H. Prov., 5,400 ft, 10. VI. 1958, fruit fly trap, Barrett (DPI) ; 2 q, Mt. Gahavisuka, 6,500 ft., 22. VII. 1983, Roberts (FRS).

Bionomics. Nothing is known.

Distribution. New Guinea (PNG).

\section{Polleniopsis fulviventris Kurahashi}

Polleniopsis fulviventris Kurahashi, 1972, Pac. Insects $14: 716$.

Length : $6.0-9.0 \mathrm{~mm}$.

SPECIMENS EXAMINED. No additional material to the type series.

BionOmiCs. Nothing is known.

Distribution. New Guinea (Irian Jaya \& PNG).

Genus Melinda Rob.-Desvoidy

Melinda Rob.-Desvoidy, 1830, Ess. Myod. : 439. Type-species : Musca cognata Meigen, 1910.

$$
\text { KEY то THE SPECIES OF M elinda }
$$

1. Third antennal segment bright orange ; wings infuscated ; tergite 3 with several erect marginal bristles on lateral sides M. ruficornis sp. nov.

- Third antenna1 segment fuscous black ; wings largely yellowish brown on basal 2/3; tergite 3 with row of erect marginal bristles M. flavipennis sp, nov. 
The following two new species seem to be an aberrant of the genus Melinda because they have a bare prosternum. The hairy propleura and prosternum are characteristic of the subfamily Calliphorinae to which the genus belongs. Besides this aberration, the general morphology of these two New Guinea species quite agrees with that of Melinda.

\section{Melinda ruf icornis, new species}

. Head : eyes hairy, dichoptic, separated at vertex by a distance equal to 0.25 of head width ; frontal stripe black, slightly narrowed posteriorly, $2.0 \mathbf{x}$ the width of 1 of parafrontalia just in front of anterior ocellus, with several pairs of interfrontal bristly hairs ; parafrontalia dark gray-dusted, with black setulae, provided with ca 4 pairs of strong ori; ors 3 , very strong, anterior one is proclinate, posterior two are directed externally ; oc developed ; acoc absent ; poc divergent ; ov and iv strong ; occ 1 strong; parafacialia dark gray-dusted, with fine black setulae above; facialia black, slightly gray-dusted, setulose on more than lower $1 / 2$; epistome and face black, submetallic ; face without median carina; medianae narrow, black, bare ; vibrissaria and genae black, submetallic, clothed with black hairs; vibrissae strong; postgenae and occiput concolorous with genae ; occiput with pale hairs on central part ; 3rd antennal segment reddish orange, slightly more than $2 \mathbf{x}$ as long as 2nd; 1st and 2nd segments black, submetallic ; arista submetallic black, long plumose on basal $2 / 3$; palpi fuscous, but reddish brown on apical $1 / 3$. Thorax : black, submetallic, gray-dusted anteriorly and laterally, one broad median, two fine submedian and one lateral broad longitudinal stripes are visible on prescutum ; median broad stripe not interrupted by transverse suture, but the other external three stripes interrupted by transverse suture or dusting on scutum; scutellum reddish apically, but largely concolorous with dorsum ; humeri and postalarcalli concolorous with thoracic dorsum, but slightly reddish on lateral sides of postalar calli; propleura blackish setulose ; other pleural hairs black; prosternum bare ; supraspiracular convexity pubescent, without erect hairs ; mesothoracic and metathoracic spiracles blackish ; pleurotergite with a patch of black setulae ; postalar declivity with a few black setulae in central circle ; a few of tympanic and anterior parasquamal hairs present. Chaetotaxy ; ac $2+3, d c 3+3$, ia $\mathbf{1}+3, h 3$, ph 2 , prs 1 , sa 3 , pa $2-3$, st $2+$ $\mathbf{1}, \mathbf{s c} 4+2,1$ pair of fine additional discals developes between apical marginals and discals, $p p$ and $p s t$ strongly developed. Wings : entirely infuscated ; veins blackish brown ; epaulet black ; basicosta blackish brown ; subcostal sclerite blackish brown, pubescent ; node of 2nd and 3rd longitudinal veins with several black setulae above and below ; 4th longitudinal vein bent with obtuse angle ; squamae blackish brown, thoracic one is lobulated, bare on upper surface. Halteres yellowish brown. Legs : black except for mid and hind tibiae slightly reddish, black haired ; front tibia with 1-2 $p$ and a row of short ad ; mid tibia with $1 \mathrm{ad}, 1 \mathrm{pd}, 2 p$ and $1 \mathrm{v}$; hind tibia with 2 ad, $2 p d$ and 2 av, besides these incomplete rows of short ad and $p d$ are also present, apical ad is well developed. Abdomen : submetallic black, reddish on venter of tergite $1+2$, lateral sides of tergite 3 and posterior margin of tergite 5, silver-gray dusted, weakly tessellated, clothed with black hairs and strong bristles ; tergite $\mathbf{1}+2$ submetallic black, with a group of long hairs on lateral sides of abdomen and a few strong lateral marginal bristles ; tergite 3 with erect lateral marginal bristles ; tergite 4 with erect strong marginal bristles ; tergite 5 with erect marginal bristles and discals. Ovipositor elongate, with corneous 7 th tergite typical of the genus Melinda.

ơ. Unknown.

Length $: 5.5 \mathrm{~mm}$.

Holotype , NEW GUINEA (PNG) : Mt. Hagen, 2,910 m, 12. II. 1978, Kano (TMDU).

Type Depository. Holotype in Bishop Museum.

Bionomics. Unknown.

Distribution. New Guinea (PNG). 


\section{Melinda flavipennis, new species Fig. 3}

$\sigma^{7}$. Head : eyes bare, holoptic, separated at narrowest point by less than the width of ocellar triangle ; frons index 0.04 ; frontal stripe black widened anteriorly and posteriorly, reduced to a fine line at narrowest point ; parafrontalia narrow, silver-gray, darkened toward vertex, with black setulae anteriorly, with ca 10 pairs of ori and fine some interstitials ; parafacialia silver-gray dusted, with fine black setulae above ; facialia black, slightly silver-gray dusted, setulose on lower $1 / 3$; epistome and face black, slightly gray-dusted; face without median carina; medianae blackish brown ; vibrissaria and genae black, gray-dusted, clothed with black hairs ; vibrissae well developed ; postgenae and occiput concolorous with genae, with black hairs ; antennae blackish; 3rd antenna1 segment, gray pubescent in a certain incident of light, slightly more than $2 \mathrm{x}$ as long as 2nd ; arista blackish brown, long plumose on basal $2 / 3$; palpi black, with black hairs. Thorax : black, covered with silver gray dusting, with dark stripes and spots more or less distinct when viewed from behind ; prescutum with two fine submedian stripes and broad triangular lateral spots, trace of median stripe is present on posterior $1 / 2$; scutum with three rather broad median and lateral stripes, trace of

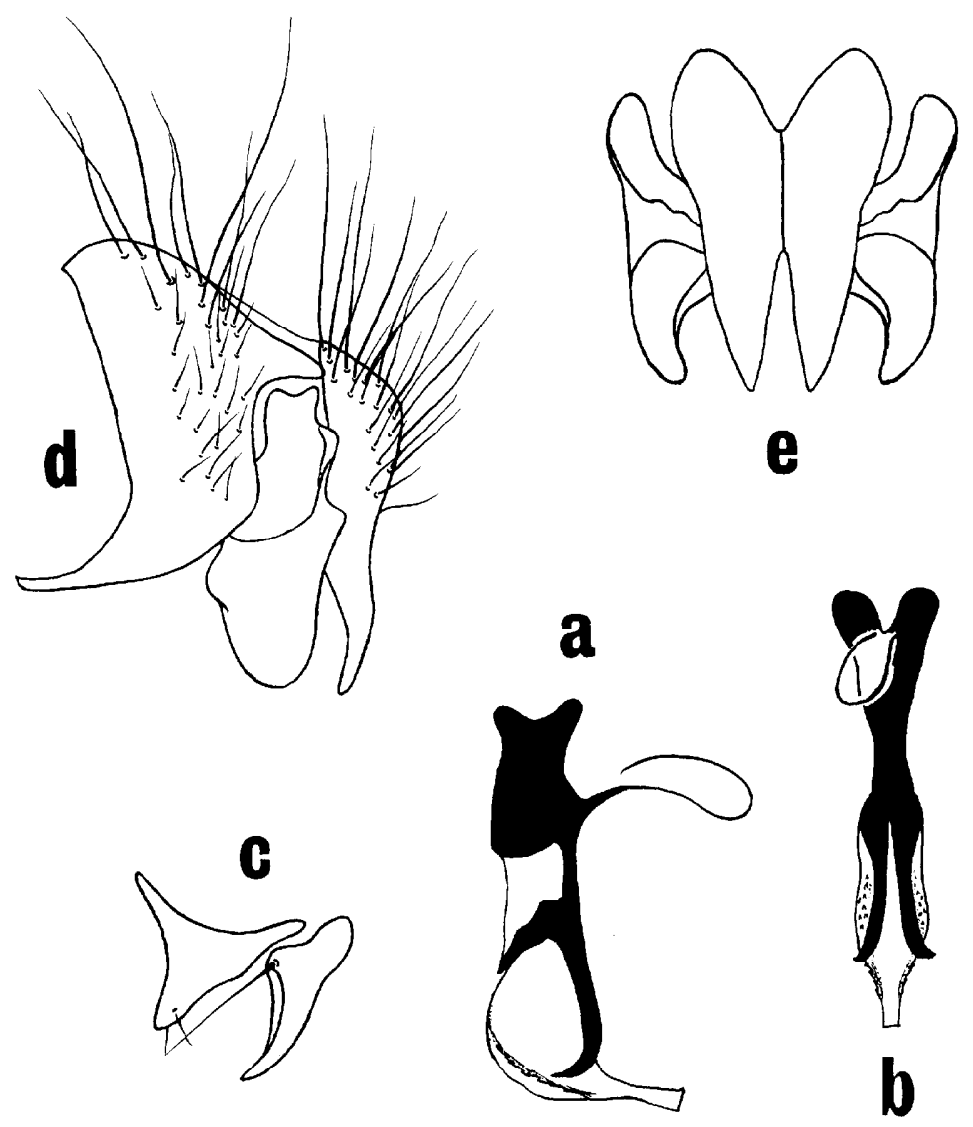

Fig. 3. Melinda flavipennis sp. nov., male hypopygium : a, aedeagus, lateral view ; b, aedeagus, posterior view; c, anterior and posterior parameres, lateral view ; d, epandrium, cercus and paralobus, lateral view ; e, cerci and paralobi, caudal view. 
submedian stripes are present on posterior $1 / 2$; scutellum, humeri and postalar calli concolorous with dorsum, postalarcalli are more or less reddish along margins; propleura with black hairs, other pleural hairs are also black; prosternum bare ; supraspiracular convexity pubescent, without erect hairs ; mesothoracic and metathoracic spiracles black; pleurotergite with a patch of black setulae ; postalar declivity with tuft of black hairs in central circle ; a few black tympanic hairs present ; no anterior parasquamal hairs present. Chaetotaxy ; ac $2+3, d c 3+3$, ia $1+3, h 3$, ph 3 , prs 1 , sa 3 , pa 2 , st $2+1$, lower 1 of anterior st is usually fine, sc $4+2$, several fine additional discals are developed between apical marginals and discals, $p p$ and $p s t$ developed. Wings : hyaline, rather strongly tinged with yellowish pigment on basal $2 / 3$, slightly infuscated on apical $1 / 3$ and posterior margin ; veins yellow ; epaulet and basicosta black ; subcostal sclerite brown, pubescent ; node of 2nd and 3rd longitudinal veins with several black setulae above and below; 4th longitudinal vein bent with a gentle curve; squamae yellowish brown, thoracic one is lobulated, without hairs on upper surface. Halteres yellowish brown. Legs : black, black haired; front tibia with $1 p$ and a row of ad on basal $2 / 3,1$ ad on apical $2 / 3$ is relatively strong; mid tibia with 1 ad, $1 p d, 2 p$ and $1 v$; hind tibia with 2 ad, $2 p d$ and $1 a v$. Abdomen : black, with brassy tinge ; gray-dusted, weakly tessellated, clothed with black hairs; tergite $1+2$ black, rather densely covered with black hairs, with strong lateral marginal bristles, besides these some additional bristles are developed on lateral sides of abdomen ; tergite 3 black, brassy, with thin covering of brownish dusting, with row of lateral marginal bristles, without median pair of marginals; tergites 4-5 black, densely gray-dusted, with weak tessellation, with complete row of erect marginal bristles, row of erect discal bristles is also developed on lateral sides. Hypopygium smaller in size, $\widetilde{O}^{\top}$ genitalia is shown in Fig. 3.

ㅇ. Unknown.

Length : $7.5 \mathrm{~mm}$.

Holotype ơ, NEW IRELAND : Namatanai, 21-23. I. 1974, Kano (TMDU). Paratype : NEW IRELAND :1 ơ', same data as holotype.

Type DEPository. Holotype in Bishop Museum ; paratype in author's collection in NSMT.

Bionomics. Unknown.

Distribution. New Ireland.

\section{Acknowledgements}

I wish to express my sincere thanks to Prof. Dr. R. Kano, Chief of the Survey, and Dr. S. Shinonaga, Tokyo Medical and Dental University ; to Prof. Dr. Y. Hirasima, Chief of the Survey, and Dr. K. Morimoto, Kyushu University ; to Mr. N. Kwapena, Office of Environment and Conservation, Boroko, PNG ; and to Mr. Allan Isoaimo, Morobe Provincial Research Commitee, Lae, PNG, who allowed and helped me to research the blow flies of the New Guinea. I am also indebted to Dr. Hywel Roberts and Mr. Tagula Siriware, Forest Research Station Bulolo, PNG ; to Dr. J. W. Ismay, Department of Primary Industry, Konedobu, PNG ; and to Dr. H. Shima, Kyushu University, Fukuo$\mathrm{ka}$, for their kind help in field surveys and in lending me valuable specimens from their collections. I am grateful, also, to Mr. Adrian C. Pont, British Museum (Nat. Hist.), London; and to the late Dr. J. L. Gressitt and the staffs of Entomology Department, Bishop Museum, Honolulu, for their valuable advice and suggestions given me during the course of study. I gratefully acknowledge Dr. Y. Wada, Director, Department of Medical Enotmology, National Institute of Health, Tokyo, for his constant encouragement. 